\title{
Evolving Musical Harmonisation
}

\author{
Somnuk Phon-Amnuaisuk, Andrew Tuson ${ }^{1}$, and Geraint Wiggins \\ Music Informatics Research Group, Division of Informatics, University of Edinburgh \\ 80 South Bridge, Edinburgh EH1 1HN, Scotland, UK. \\ Email: \{somnukpa,andrewt,geraint $\}$ dai.ed.ac.uk
}

\begin{abstract}
We describe a series of experiments in generating traditional musical harmony using Genetic Algorithms. We discuss some problems which are specific to the musical domain, and conclude that a GA with no notion of meta-level control of the reasoning process is unlikely to solve the harmonisation problem well.
\end{abstract}

\section{Introduction}

In recent years, the use of evolutionary techniques such as Genetic Algorithms (GAs) has generated significant interest in the artificial intelligence and computer science communities. This has been reflected in a number of publications in the computer music world, some of which will be discussed later.

In this paper, we explore two aspects of the application of GAs to music:

1. the use of knowledge-rich structures and procedures within the algorithm itself, as opposed to the more traditional use of GA components which are not problem-specific;

2. the strict use of objective methods, in the sense that any reasoning encoded in the GA should be stated explicitly, rather than being implicit in the expressed opinion of a human user.

These criteria are important because we are working in the wider context of simulating and understanding aspects of human behaviour, so we are not interested just in achieving a musical result: we wish to be able to examine the internal behaviour of our methods, and attempt to form some notion of why the answer we achieve is produced. In particular, we wish to compare the behaviour of our harmonisation system with human behaviour, and attempt to explain any discrepancies.

This paper is structured as follows. We present a brief statement on the issues interaction $v s$. noninteraction in GAs from the point of view of this study. We then outline existing applications of GAs in computer music. We present a case study of a knowledge-rich musical GA, including a discussion of some significant problems, and then draw conclusions about the implications of the work for musical GAs in general.

\section{Interactive GAs}

The Interactive GA (IGA) approach, sometimes taken by GA applications in the musical domain (see section 3, below), uses a human listener as a means to evaluate the fitness of chromosomes. This approach is inappropriate to our ends in this research, for the following reasons.

\footnotetext{
${ }^{1}$ Andrew Tuson may now be contacted care of the Department of Computing, City University, London, UK.
} 
1. It is subjective, because it relies on individual preferences - we want an objective measure of what is going on in our system, so we can properly judge its performance;

2. Human listeners tend to become more open to a given piece of music on repeated hearings, and are prone to other inconsistencies based on mood, attention span, and so on;

3. An IGA does not allow us to study the fitness function itself, to determine how faithful it is to our chosen task - so using an IGA would be removing a major object of interest in this study.

A further tenable position on this issue, lying part-way between the two poles of interactive and non-interactive, is the idea of using a corpus of existing works and some form of machine learning system to infer a fitness function. This approach has been applied by, for example, Spector and Alpern (1994), Burton and Valdimirova (1997b) and Johanson and Poli (1997). Again, for our purposes, this approach is inappropriate, as we are primarily interested in the nature of the search space and how to control search, rather than the nature of the result (given that it be acceptable music). In fact, use of neural networks makes the fitness judgements even more inscrutable, since at least one can ask a human judge for the motivation behind his or her judgements.

In the experiments described here, music-theoretical knowledge is used to construct a fitness function in objective and consistent logical terms, which allows us to examine the behaviour of the system more scientifically than would an IGA. The GA can then be used to compare theories (psychological or otherwise) of musical behaviour by observing the search patterns and results produced. In particular, it is to be emphasised that the encoded musical knowledge does not directly constrain the search path - it merely constrains the solution. So our interest focusses on what our GA can tell us about the search paths which arise from this unconstrained setup.

\section{Existing Work on GAs in Music}

GAs have been used in music generation elsewhere. Examples include Horner and Goldberg (1991) who used a GA for thematic bridging; Biles (1994), who used an IGA for Jazz improvisation; Jacob (1995), who devised a composing system using an IGA; and McIntyre (1994) and Horner and Ayers (1995). McIntyre used a GA to generate a four part harmonisation of an input melody, focusing on Baroque harmony, while Horner and Ayers focussed on the harmonisation of chord progressions using GAs.

A main aim of our harmonisation project is to investigate the potential of a knowledge-rich GA and its performance in the musical domain. So our solution space is not artificially constrained as in McIntyre's system (which only used a $\mathrm{C}$ major scale); nor is there problem abstraction as in Horner and Ayres' system, (which uses the GA to generate parts, given a chord progression, which is a significantly simpler task). Our work aims to harmonise input melodies with no explicit cues as to the required harmony, and does not limit itself to a specific key or scale; and it works at the level of individual voices, with all the extra constraints this entails.

Finally, for a more complete summary of GA work in music, see Burton and Vladimirova (1997a).

\section{Harmonising Chorale Melodies by GA}

In this section, we present the results of a study on the use of GAs in generating four-part homophonic tonal harmony for user-specified melodies. The domain-specific (i.e., musical) knowledge in this system is implemented in three parts of the GA. These are described in turn, and followed by an overview of the GA configuration used. 


\subsection{Chromosome Representation}

Generally speaking, keys and chords are the main concepts in harmonisation of western tonal music. Harmonisation rules are expressed in terms of relationships between triads, and between degrees of scale within a key signature (e.g., tonic-dominant, etc) but not the absolute pitch. Therefore, in this implementation, musical information (e.g., pitch, interval, time, duration) is represented after normalisation with respect to key - that is, absolute pitch information is abstracted out. Then, pitch is expressed in terms of scale degree. To express all twelve semitones, the standard five accidentals are used. Different octaves are distinguished by an associated integer. Finally, time intervals are represented as integers. The representation conforms to the CHARM specification of Wiggins et al. (1989).

As noted earlier, a knowledge-rich and directly meaningful representation is used in our chromosome representation. This representation may be thought of as a matrix, which consists of five strings of equal, fixed length. The top four strings contain soprano (fixed), alto, tenor and bass parts, with the fifth describing the durations of the chords. The user inputs the soprano information (assumed to be the melody); the GA will then harmonise the input soprano, homophonically, with a further three voices in conjunction with the musical domain knowledge encoded in its operators and fitness function.

This approach is illustrated in Figure 1, using the completed harmonisation for the first two bars of "O Come, All Ye Faithful"; see Figure 4 for the score rendition of the corresponding output.

chromosome length

\begin{tabular}{|c|c|c|c|c|c|c|}
\hline Soprano & {$[0,0,3]$} & $\overline{[0,0,3]}$ & $\overline{[4,0,2]}$ & {$[0,0,3]$} & $\overline{[1,0,3]}$ & {$[4,0,2]$} \\
\hline Alto & {$[2,0,2]$} & {$[2,0,2]$} & {$[2,0,2]$} & {$[2,0,2]$} & {$[4,0,2]$} & {$[1,0,2]$} \\
\hline Tenor & {$[4,0,1]$} & {$[4,0,1]$} & {$[2,0,1]$} & {$[0,0,2]$} & {$[7,0,1]$} & {$[1,0,1]$} \\
\hline Bass & {$[2,0,1]$} & {$[0,0,1]$} & {$[0,0,1]$} & {$[4,0,1]$} & {$[4,0,1]$} & {$[7,0,0]$} \\
\hline uration & $\overline{1}$ & $\overline{2}$ & $\overline{1}$ & $\overline{1}$ & $\overline{2}$ & $\overline{2}$ \\
\hline
\end{tabular}

Figure 1: Schematic Diagram of a Four-Voice Harmony Chromosome

In the figure, we can see the matrix arrangement produced by the direct representation of time (left to right along the structure) and vocal part and note duration (top to bottom down the structure). For the purposes of reproduction operators, we view the five horizontal layers as inseparable.

\subsection{Reproduction Operators}

The following crossover and mutation operators are used in this implementation, described here in musical terms. The reader less familiar with musical jargon may find solace in Taylor (1996).

Splice: One point crossover between two chromosomes - selects a crossover point between successive notes of the melody and corresponding chords.

Perturb: Mutate by allowing alto, tenor and bass to move up or down by one semitone or tone. The selection of the various possible mutations is random.

Swap: Mutate by swapping two randomly picked voices between alto, tenor or bass. This gives the effect of changing the chord between different open and closed positions, and of changing inversions.

Rechord: Mutate to a different chord type. This mutation generates a new chord from the melody data. A chord is built with the soprano note as root, 3rd or 5th. Doubling (necessary for a four note chord) can be in any position. 
PhraseStart: Mutate the beginning of each phrase to start with tonic root position on a down beat.

PhraseEnd: Mutate the end of each phrase to end with a chord in root position.

\subsection{Fitness Function}

The fitness function judges the fitness of each chromosome according to the following criteria derived directly from music theory. Within individual voices (as opposed to between voices), we prefer stepwise progression over large leaps, and we keep the voice within its proper range. We penalise progression to dissonant chords, and we avoid leaps of major and minor 7ths, of augmented and diminished intervals, and of intervals larger than one octave.

Between voices, we apply the following criteria: we avoid parallel unison, parallel perfect 5ths, and parallel octaves; we forbid progression from diminished 5th to perfect 5th (though the converse is allowed); we avoid hidden unison; we forbid crossing voices; and we forbid hidden 5th and octave in the outer voices, when soprano is not progressing stepwise.

Solutions are penalised for note doubling and omission, in the primary major and minor triads: doubling of the root (tonic) is preferred, while doubling of the 3rd is penalised; doubling of the 3rd is forbidden in a dominant chord; if it is necessary to omit a voice, omit the fifth only, except in 1st inversion; in inverted chords, doubling of the bass is preferred; and we penalise doubling of tones which give a strong harmonic tendency, such as leading notes.

In this implementation, the system does not have enough knowledge to plan for large scale harmonic progression. The fitness function determines only the plausible harmonic movement between two adjacent chords. The fitness function prefers (in decreasing order of preference): descending 5th movement; progression towards the tonic; retrogression; and repetition.

\subsection{GA Configuration}

In most of these experiments, exceptions being mentioned explicitly here, a generational GA, in the style of Davis (1991), with a panmitic (unstructured) population model was used. Strings were initialised by randomly picking chords containing the relevant soprano pitch. Finally, a population size of at least 50 was used, with binary tournament selection.

\section{Results and Analysis}

All the output of the system was assessed by Dr. John Kitchen, a senior lecturer in the Faculty of Music at the University of Edinburgh, according to the criteria he uses for 1st year undergraduate students' harmony. This example scored 5 out of 10 - a clear pass. While other examples were less successful (most earning around the $30 \%$ mark), according to the assesser, this was mostly due to the lack of coherent large-scale musical progression - which was not evaluated in the fitness function, so this is not a surprise. The system was judged by the assesser to be better than student harmonisers at getting the basic rules right.

Figure 2 shows a harmonisation by our system of the first eight notes of "Joy to the world". The output is not perfect, but it is surprisingly good given the limited, local nature of the rules built into the system.

Some experimentation was carried out with various GA parameter settings, as shown in Figure 1; the penalties given here are all-or-nothing penalties, except where two numbers are given, in which case either value may be applied as appropriate. As expected, the weights of the various penalties applied in the fitness function have a significant effect on the solution. Other parameters, such as crossover rate, mutation rate, and different selection schemes appear to affect the time taken for the population to converge, and do little for the solution quality. This is due to the fact that it is the fitness 


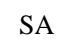

TB

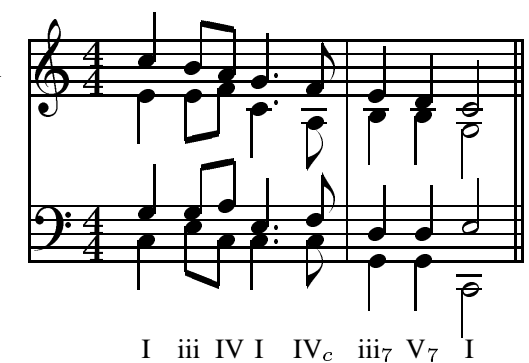

Figure 2: Harmonisation of the First Line of Joy to the World

function which primarily defines the knowledge in the system pertaining to what does or does not constitute a good piece of music, while the other parameters define the search strategy.

Table 1: Probabilities and penalties used in the experiments

\begin{tabular}{|lc|lc|}
\hline Population: & $30-200$ & Migration interval: & 20 generations \\
\hline \multicolumn{4}{c|}{ Operator Probabilities } \\
\hline P(Splice): & 0.3 & P(Perturb): & 0.1 \\
P(Swap): & 0.2 & P(Rechord): & 0.3 \\
P(PhraseStart): & 0.05 & P(PhraseEnd): & 0.05 \\
\hline \multicolumn{4}{|c|}{ Fitness Penalties } \\
\hline Invalid Pitch: & 1 & Invalid Chord: & 10,1 \\
Invalid Range: & 10 & Invalid Interval: & 10 \\
Invalid Doubling: & 10 & Voice Crossing: & 10 \\
Hidden Unison: & 10 & Single voice progression: & 10 \\
Dual voice progression: & 10 & Harmonic progression: & 10,1 \\
Harmonic Analysis: & 100,20 & & \\
\hline
\end{tabular}

What is most significant - and problematic - is that, with the current evaluation functions and reproduction operators, the GA still cannot satisfy all the constraints within 300 generations. Figure 3 illustrates a typical fitness profile of the best solution in each generation. The data is from the first phrase of the hymn "O come, all ye faithful", which is twenty four notes long. The figure shows the distribution of penalties along the chromosome - in other words, the score of each harmonic movement or chord in the piece - as the generations proceed. The higher the contour, the less acceptable the chord at that point.

In the figure, we can see that the distribution of penalties at the beginning is quite random. After a few generations, it starts to shape to a certain pattern. However, the GA could not reduce all the penalties in the fitness function even with enlarged populations and when run for large amounts of time. In an attempt to solve this problem, an experiment with an island model (Gordon et al., 1992) with four population groups was carried out, to determine whether different groups might be able to preserve their own salient cultures, and so bring the GA to a more globally acceptable solution. However, though the experiment showed an improvement in search efficiency, the GA still could not reduce all of the penalties at once, and the general shape of the unacceptable contour above was maintained. The musical output associated with that final contour is shown in Figure 4.

Why, then, do these problems arise? They arise because the structure of a harmonisation of this kind is very specific, in that individual variations in chord are very strongly context dependent. Therefore, it is often the case that one cannot change any given chord without changing the chords around it. In terms of the GA, this means that reduction of a fitness penalty in one position is likely to increase penalties in other positions, because the movement from one chord to the next is not considered 


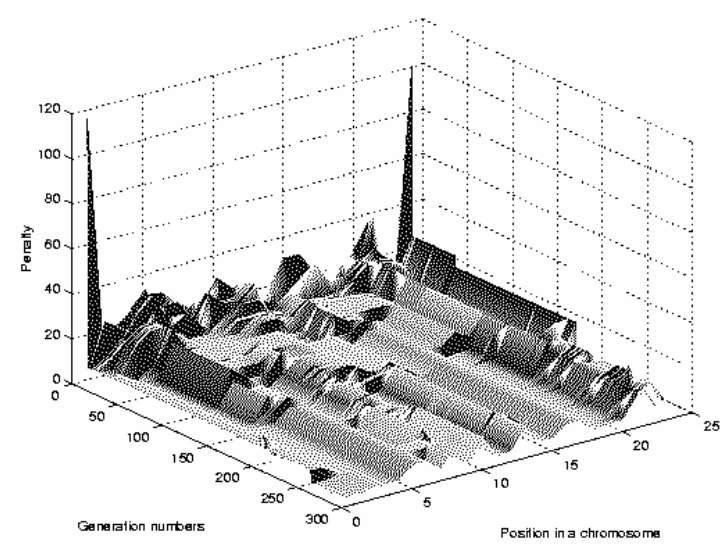

Figure 3: A Typical Fitness Profile Landscape

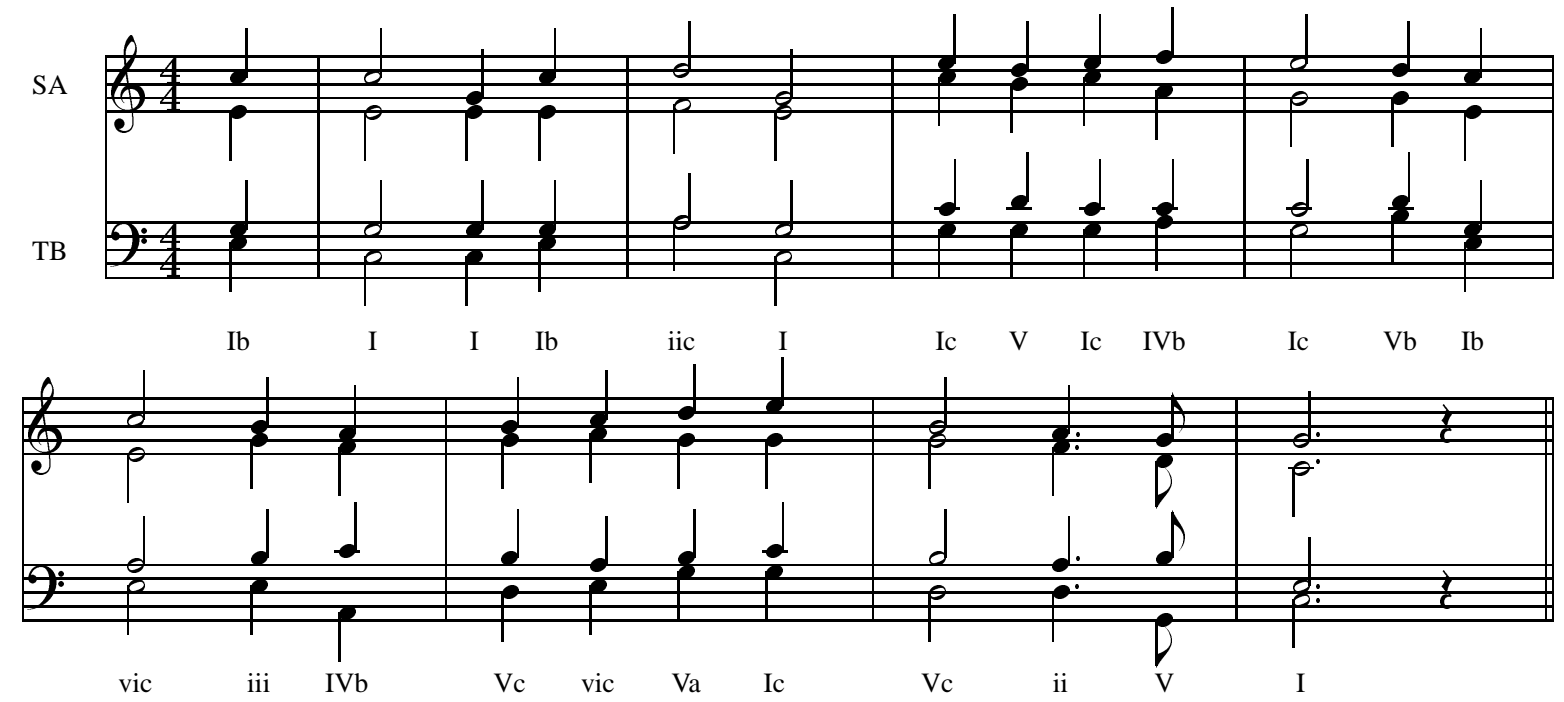

Figure 4: A GA Harmonisation of "O Come, All Ye Faithful"

with respect to overall movement in the phrase. Human composers solve this problem by structuring the construction process itself in an explicit way and designing an overall harmonic framework, and then filling in the most crucial parts first - in short, a least commitment strategy is taken. This kind of structured reasoning is not a feature of a simple GA of the kind used here, and so the results are sub-optimal.

To restate this argument in terms of the search space: we believe that the problem is due to a multimodal fitness landscape, characterised by many local optima in basins of attraction which separated by very high barriers, due to the interactions described above. Before the GA can move from one basin of attraction to another, multiple factors leading to a fitness penalty need to be changed. Such a simultaneous change is very unlikely to occur.

There are various solutions which might be applied to solve this problem in a GA context. Niching (Goldberg and Richardson, 1987) might be expected to help with the problems of multimodality, and we expect to look at this in future. Linkage learning (Harik and Goldberg, 1996) is unlikely to help as the spatial arrangement of the genes in our chromosome is already optimised, and so changing it is unlikely to help the search. Further, the nature of the epistatic interactions is well understood, and therefore this knowledge can be better utilised directly, rather than by having the GA learn it. 


\section{Conclusions}

It is quite clear from the experiments here and elsewhere that GAs can be applied successfully in the musical domain - up to a point. Looking at the output of our systems from an aesthetic viewpoint, the results are still far from ideal: the harmonisation produced by the GA has neither clear plan nor intention. This is not a surprise as the discussion above suggested that we cannot expect large scale structure to arise from the kind of programming inherent in a GA containing (even in this experiment) relatively little domain knowledge.

However, we would claim that they are surprisingly successful musically, within this limitation. The inclusion of well-established musical laws and constraints within the search seems to yield a fairly lightweight but effective level of search control.

In summary, therefore, we conclude that while GAs can be surprisingly good at small, constrained musical tasks, their performance, at least in this context of simulating human musical behaviour, is currently limited by two issues.

1. GAs are a stochastic, heuristic search method, so one cannot be sure that an optimal solution will be reached, even if there is one. In particular, in a problem of this kind, they tend to get stuck in local optima in the search space.

2. GAs of the form used here lack structure in their reasoning. On the other hand, composers have developed complex and subtle methods over several centuries involving different techniques for solving the problems highlighted here. No musician would seriously suggest that an author of hymn tunes works in the same way as our GA. Therefore, while we may be able to produce (near) acceptable results with a GA, doing so says little about the working of the compositional mind.

Other approaches to using GAs for this kind of task may be more successful. For example, one might take the approach of optimising a set of instructions to plan a harmonisation, rather than actually operating on the musical score directly; this approach is similar to the indirect representational approach used by Burke (1995) and others for timetabling and scheduling problems.

Genetic programming approaches may also be fruitful, but we suggest that in order to produce music which is coherent within any accepted musical system (e.g., the tonal system used in the vast majority of rock and pop music), there will need to be some encoding of musical practice in the GP operators. An example of a GP music system which suffers from lack of such knowledge is the GP-music program of Johanson and Poli (1997).

An alternative solution in the context of our GA would be to introduce even more knowledge-rich mutations, which would possess knowledge about the entire harmonic structure of each candidate solution, and thus would be able to leap directly across the barriers in the fitness landscape described earlier in a single bound. However, it would be hard indeed to make a clear differentiation between this approach and a conventional KBS, if indeed one could apply such a rule without being unacceptably ad hoc.

We conclude, therefore, that neighbourhood search methods such as GAs are fundamentally limited in the musical harmonisation domain by the non-local nature of the harmonisation problem itself. This said, at least intuitively, GAs seem to offer an interesting approach to the study of creativity. It would appear that, if GAs are to both improve in musical performance, and allow us to gain insights into the compositional mind, dealing with the issues raised here is an urgent task. We end with a suggestion that it is likely that much can be gained in this particular problem by somehow combining a GA with a conventional rule-based system. 


\section{Acknowledgements}

Thanks to Dr. John Kitchen for his help in assessing the harmonisation system. Andrew Tuson is supported by EPSRC studentship, reference number 95306458 .

\section{References}

Biles, J. A. (1994). GenJam: A genetic algorithm for generating jazz solos. In ICMC Proceedings 1994. The Computer Music Association.

Burke, E., Elliman, D., and Weare, R. (1995). The Automated Timetabling of University Exams using a Hybrid Genetic Algorithm. In The AISB Workshop on Evolutionary Computing.

Burton, A. R. and Vladimirova, T. (1997a). Applications of genetic techniques to musical composition. Available by WWW at http://www.ee.surrey.ac.uk/Personal/ A. Burton/work.html.

Burton, A. R. and Vladimirova, T. (1997b). A genetic algorithm for utilising neural network fitness evaluation for musical composition. In Proceedings of the 1997 International Conference on Artificial Neural Networks and Genetic Algorithms, pages 220-224.

Davis, L., editor (1991). Handbook of Genetic Algorithms. New York: Van Nostrand Reinhold.

Goldberg, D. E. and Richardson, J. (1987). Genetic algorithms with sharing for multimodal function optimization. In Proceedings of the Second International Conference on Genetic Algorithms and Their Applications, pages 41-49. San Mateo: Morgan Kaufmann.

Gordon, V., Whitley, D., and Böhn, A. (1992). Dataflow parallelism in genetic algorithms. In Männer, R. and Manderick, B., editors, Parallel Problem Solving from Nature 2, pages 553-42, Amsterdam. Elsevier Science.

Harik, G. R. and Goldberg, D. E. (1996). Learning linkage. In Foundations of Genetic Algorithms IV, pages 270-85. San Mateo: Morgan Kaufmann.

Horner, A. and Ayers, L. (1995). Harmonisation of musical progression with genetic algorithms. In ICMC Proceedings 1995, pages 483-484. The Computer Music Association.

Horner, A. and Goldberg, D. E. (1991). Genetic algorithms and computer-assisted music composition. Technical report, University of Illinois.

Jacob, B. L. (1995). Composing with genetic algorithms. Technical report, University of Michigan.

Johanson, B. and Poli, R. (1998). Gp-music: An interactive genetic programming system for music generation with automated fitness raters. In Proceedings of the 3rd International Conference on Genetic Programming, GP'98. MIT Press.

McIntyre, R. A. (1994). Bach in a box: The evolution of four-part baroque harmony using a genetic algorithm. In First IEEE Conference on Evolutionary Computation, pages 852-857.

Spector, L. and Alpern, A. (1994). Criticism, culture, and the automatic generation of artworks. In Proceedings of the 12th National Conference on Artificial Intelligence.

Taylor, E. (1996). The AB Guide to Music Theory. The Associated Board of the Royal Schools of Music, London. 
Wiggins, G. A., Harris, M., and Smaill, A. (1989). Representing music for analysis and composition. In Balaban, M., Ebcioglu, K., Laske, O., Lischka, C., and Sorisio, L., editors, Proceedings of the 2nd IJCAI AI/Music Workshop, pages 63-71, Detroit, Michigan. Also from Edinburgh as DAI Research Paper No. 504. 